\title{
EL SISTEMA DE POLJES DE LA REGIÓN DE DYSTOS-ALMYROPOTAMOS (ISLA DE EUBEA, GRECIA): CARTOGRAFÍA Y EVOLUCIÓN GEOMORFOLÓGICA*
}

\author{
J. L. Peña-Monné(1), M. de Dapper(2), A. Constante ${ }^{(1)}$, \\ B. de Vliegher ${ }^{(2)}$ y K. Pavlopoulos ${ }^{(3)}$ \\ (1) Dpto. de Geografía y Ordenación del Territorio. \\ Universidad de Zaragoza, 50009. Zaragoza (España). \\ jlpen@unizar.es anaconst@unizar.es \\ (2) Dpt. of Geography. Ghent University. Krijgslaan, 281 (S8). B-9000 Gent (Bélgica) \\ morgan.dedapper@UGent.be beata.devliegher@rug.ac.be \\ (3) Dpt. of Geography. Harokopio University. 70 El. Benizelou St. \\ 17671 Athenas (Grecia) kpavlop@hua.gr
}

\begin{abstract}
Resumen: Se presenta la cartografía de la zona kárstica desarrollada sobre mármoles y esquistos del sector central de la isla de Eubea. Destaca en primer lugar el sistema de poljes, relacionados con la estructura geológica y la evolución climática de la isla durante el Cuaternario. De los 12 poljes diferenciados, tres de ellos se mantienen todavía en situación cerrada. Estas depresiones presentan extensos desarrollos de superficies de aplanamiento y conos rocosos generados por procesos de criptocorrosión kárstica en diferentes momentos de su evolución cuaternaria. Por otra parte, las áreas de cumbre aparecen con restos de superficies aplanadas heredadas, afectadas por campos de lapiaz y dolinas. Se propone un modelo evolutivo y se relaciona con otras áreas kársticas del ámbito mediterráneo.
\end{abstract}

Palabras clave: Grecia, Karst, poljes, Cuaternario.

\begin{abstract}
A geomorphological mapping of a karstic area developed on marbles and schists in the central sector of the island of Eubea is presented. The system of poljes constitutes the main geomorphological feature. It is related both to the geological structure and to the climatic evolution during the Quaternary. Only three out of the 12 poljes differentiated remain in closed conditions. These depressions display extensive planation surfaces and cones rocheux generated by karstic cryptocorrosion processes during different stages. On the other hand,
\end{abstract}

* Recibido: 29-02-08. Aceptado: 17-06-08. 
remnants of older planation surfaces affected by karren and dolines have been identified in the summit areas. Finally, the proposed geomorphological evolutive model is related to other Mediterranean karstic areas.

Keywords: Greece, Karst, poljes, Quaternary.

\section{Introducción}

Entre las morfologías kársticas existentes en el ámbito mediterráneo ocupan un papel muy destacado, por su extensión y abundancia, las depresiones fluviokársticas de tipo polje. Su estudio ha tenido especial importancia en el karst dinárico, Peloponeso, sur de Francia y algunas áreas de la Península Ibérica, como las Béticas y la Cordillera Ibérica. Su interés radica en su organización en sistemas, a veces interconectados entre sí, y en la presencia de elementos evolutivos que permiten aproximarse desde la morfología kárstica a la reconstrucción paleoambiental del Cuaternario.

El objetivo del trabajo ha sido la realización de una cartografía geomorfológica, centrada en las formas kársticas de la región de Dystos-Almyropótamos, que sirvió de punto de partida para el posterior estudio de campo. Se han utilizado fotografías aéreas a escala 1:40.000 del año 1988 y se dispuso de cartografía topográfica y geológica a escala 1:50.000.

Entre los temas de interés estaba la definición de los límites de los paleopoljes que forman la región y el establecimiento de sus características evolutivas y morfológicas; por otra parte interesaba definir la relación de estas depresiones con el dispositivo estructural complejo de la isla. Además, la existencia de morfologías kársticas del mismo estilo en otras zonas de Grecia (Bonnefont, 1972; Dufaure, 1975, 1985; Faugères, 1978; Papadopoulos-Vrynioti, 2004) y la posibilidad de compararlas también con morfologías de poljes analizados en la Península Ibérica y otros puntos del Mediterráneo, permitía comprobar la influencia de factores idénticos en su desarrollo, aunque con algunas diferencias de detalle en su evolución. En la misma isla de Eubea, se han descrito otros sistemas kársticos, como el semipolje de Seta (sector central de la isla) (Papadopoulos-Vrynioti y Kirdis, 1995) o los poljes de la península de Lichas, en el extremo NW de Eubea (Papadopoulos-Vrynioti, 1998-1999, 2004).

\section{El área de estudio}

La isla griega de Eubea está situada en el Mediterráneo oriental, al Este de la península del Ática y al sur de la región de Tesalia, quedando separada de la 
Península helénica mediante un estrecho paso del mar Egeo (Fig. 1). La isla es muy montañosa, con alturas que superan los $1000 \mathrm{~m}$ en la mayor parte de su extensión, destacando el macizo central de Dirfus, con 1743 m y, en el extremo meridional, el Monte Ochi, con $1398 \mathrm{~m}$. En su interior hay también depresiones abiertas en materiales blandos, así como grandes cuencas kársticas, destacando la de Dystos, cuyo fondo está ocupado por un lago, a tan sólo $18 \mathrm{~m}$ de altura s.n.m.

La región kárstica de Dystos-Almyropótamos está situada en el sector meridional de la isla, donde adopta forma estrecha y alargada y dirección prácticamente meridiana. Las alturas máximas (300-600 m.s.n.m.) corresponden a las cumbres relativa-

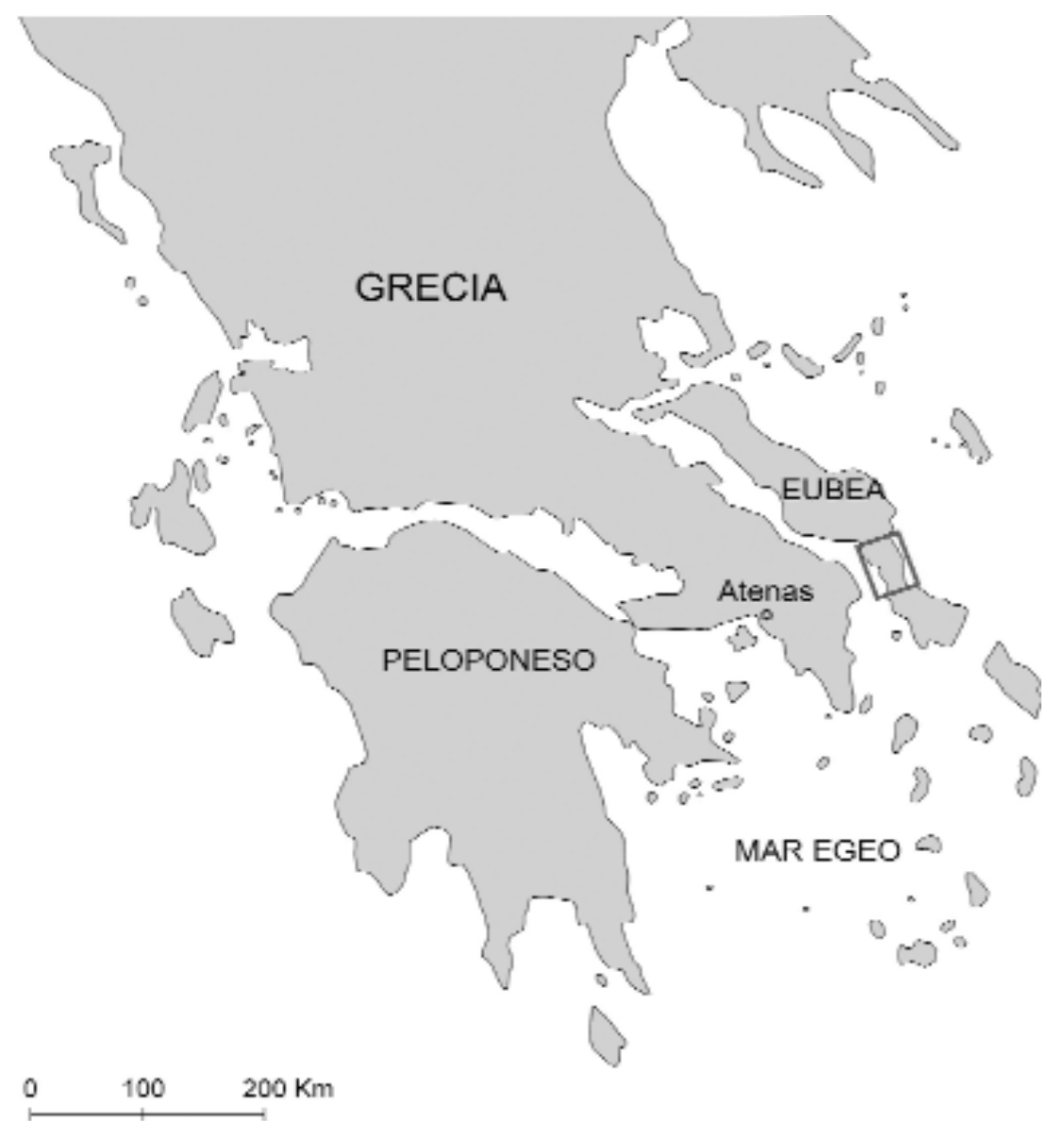

Figura 1. Mapa de situación. 
mente aplanadas de los macizos kársticos que bordean las depresiones de tipo polje. La apariencia montañosa se ve potenciada por la fuerte incisión de la red fluvial y los abruptos acantilados que los ponen en contacto con el mar. La costa está muy recortada, con numerosos entrantes, destacando el Golfo de Almyropótamos, en la costa occidental.

Desde el punto de vista geológico, el sector sur de la isla de Eubea forma parte del Macizo Cicládico en un área de intensa actividad tectónica durante el Eoceno medio. La región de Dystos puede dividirse en dos sectores, que pertenecen a dos unidades tectónicas diferentes (Andronopoulos, 1991) (Fig. 2):

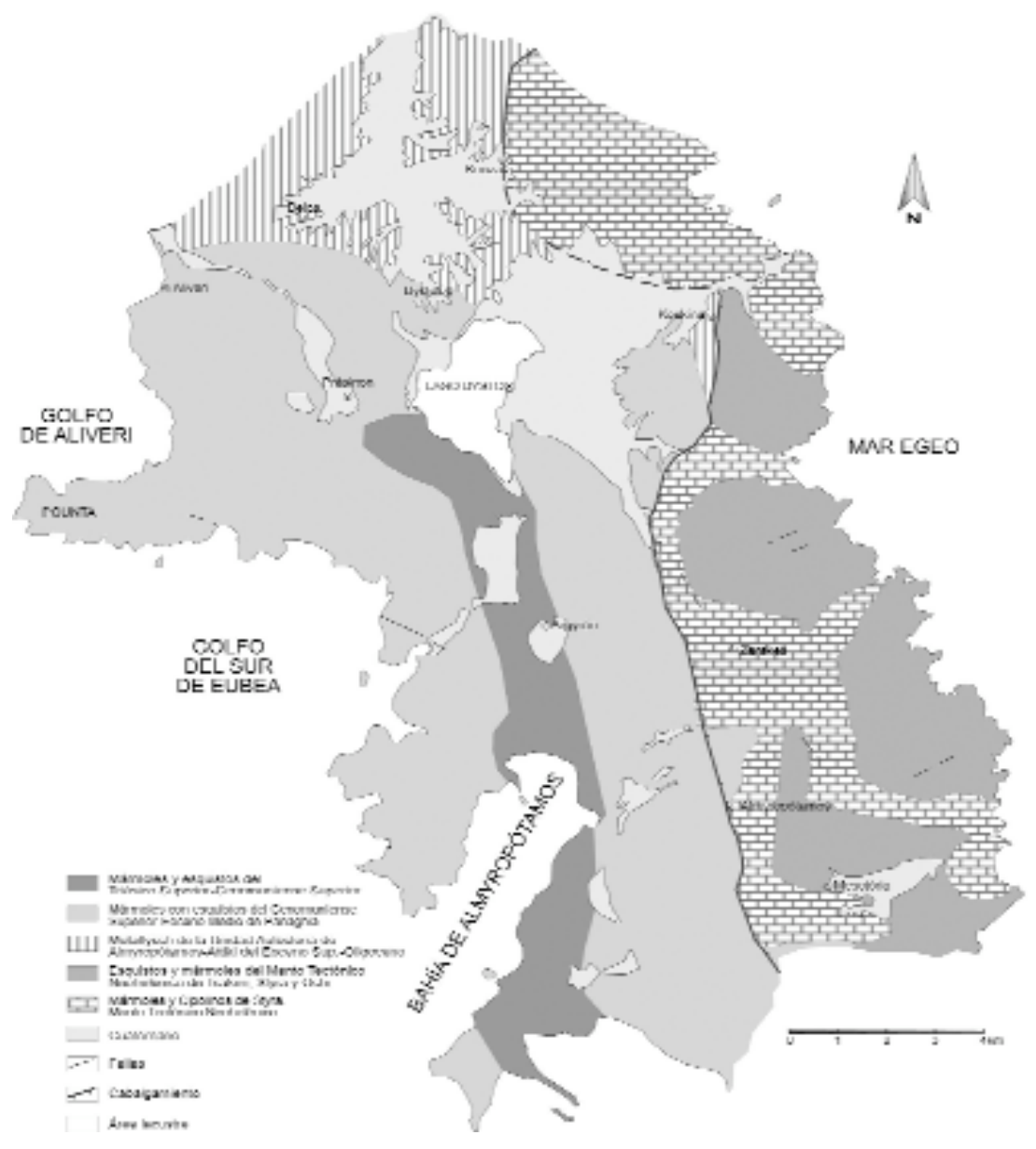

Figura 2. Unidades geológicas de la región de Dystos-Almyropotamos. 


\subsection{Unidad Autóctona de Almyropótamos-Ática}

Ocupa la parte occidental de la isla de Eubea y se compone de tres formaciones:

a) Los mármoles con intercalaciones de esquistos de Agios Giorgios-Dystos, pertenecientes al Triásico Superior-Cenomaniense Superior, que afloran en una franja estrecha, orientadas de $\mathrm{N}$ a $\mathrm{S}$ estructuralmente constituyen el núcleo de un anticlinal de eje meridiano, bordeado por flancos de la siguiente formación, entre el lago de Dystos y el S de la bahía de Almyropótamos.

b) Los mármoles de Panaghia, con niveles de esquistos, del Cenomaniense superior-Eoceno medio, que ocupan los relieves montañosos del litoral occidental y del centro de la isla, también alineados de N. a S.

c) El metaflysch esquistoso con capas calizas cristalinas del Eoceno superiorOligoceno, en el extremo Noroeste.

\subsection{Manto Tectónico Neohelénico}

Corresponde al sector oriental de la isla y cabalga sobre el Autóctono de Almyropótamos-Ática, siguiendo una línea NNE-SSE. Se compone de dos formaciones litológicas de edades posteriores al Eoceno medio:

a) Los esquistos de Tsakeoi, Styra, Panaghia y Ochi.

b) Los mármoles y cipolinos de Styra, ocupando las zonas más elevadas del macizo montañoso.

Estructuralmente, el accidente más importante corresponde a la línea de cabalgamiento del Manto Neohelénico, que se alarga de Norte a Sur por la parte central de este sector de la isla, de manera que la parte oriental se compone de materiales más antiguos, aunque no hay grandes diferencias litológicas con respecto al sector centrooccidental de Dystos. Esta zona presenta una macroestructura anticlinal, en cuyo eje afloran los mármoles y esquistos mesozoicos (Fig. 2).

\section{Organización general del sistema de poljes}

Las depresiones de tipo polje son consideradas formas de origen fluviokárstico, es decir, generadas por una combinación de procesos, en parte kársticos y en parte debidos a la escorrentía superficial. El término polje procede del karst dinárico y fue definido por Cvijic (1895; en Gams, 1978) como una gran depresión de fondo plano y 
con alargamiento paralelo a las estructuras locales. A esto habría que añadir la presencia de bordes abruptos (Monroe, 1970) y un fondo relleno de material aluvial y arcillas residuales de la disolución. El drenaje dominante es subterráneo, ya que son depresiones normalmente secas, excepto en momentos de fuertes lluvias, en los que puede llegar a inundarse (Gèze, 1973), como ocurre en el polje de Dystos (Fot. 1) y otros poljes del karst dinárico; en ocasiones hay verdaderos cursos de agua circulando en su interior, que pueden llegar a incidir profundamente, como ha ocurrido en el polje de Almyropótamos. Los fondos de estos poljes constituyen zonas agrícolas de gran importancia, dadas las escasas posibilidades que ofrecen los macizos montañosos entre los cuales se ubican.

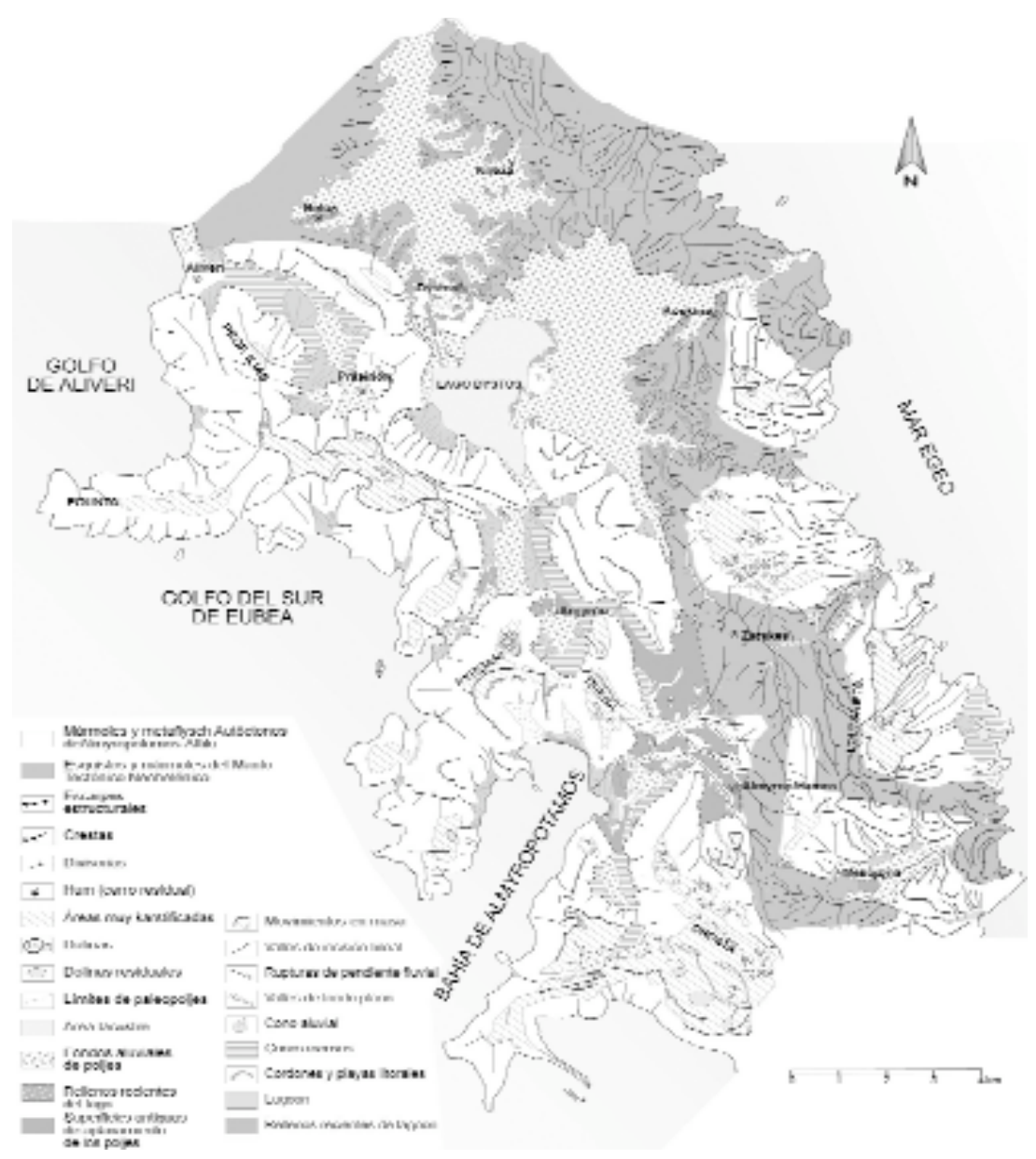

Figura 3. Mapa geomorfológico general. 
La cartografía geomorfológica (Fig. 3) muestra la distribución espacial del conjunto de poljes, que hemos organizado para su análisis en once unidades, cuyas características de dimensiones, tipo de drenaje, orientación y profundidad aparecen reflejadas en la Tabla 1.

Los poljes de mayor tamaño son los de Dystos $(7,7 \times 6,3 \mathrm{~km})$, Krieza $(>6,7 \times 5,1$ $\mathrm{km})$ y Almyropótamos (4,7 x 1,8 km), aunque incluso los de menores dimensiones superan el kilómetro de longitud y los $400 \mathrm{~m}$ de anchura, de manera que entrarían de lleno en las medidas límite propuestas por Gams (1978) para los poljes del karst dinárico, que establece su fondo plano en $400 \mathrm{~m}$ como mínimo. Los poljes ocupan depresiones profundas, ya que frente a los 300-600 m de los macizos montañosos que forman las divisorias, sus fondos están por término medio en unos 40-60 m de altura. Los situados en posición más alta son los poljes de Argyron y Lioutriza, cuyos fondos está a unos 200 m.

Otra característica general de estos poljes es la de haber perdido su morfología cerrada original y estar abiertos al drenaje fluvial general, exceptuando los de Dystos, Argyron y Lioutriza, que todavía permanecen cerrados. Estas aperturas son consecuencia de la captura de la red fluvial, seguramente debido a la cercanía y profundidad del nivel de base marino, aunque en muchos casos es la acción directa del mar la que ha provocado no sólo la apertura sino también el retroceso y pérdida de superficie de la depresión kárstica. Así ocurre con los poljes de Zarakes, Purgari y Prasinon, en los que los entrantes costeros delatan la antigua continuidad de los poljes a través de los cuales se prolongan; incluso, la Bahía de Almyropótamos, por su morfología y disposición podría ser la continuación del actual polje abierto de ese mismo nombre.

Tabla 1. Principales parámetros de los poljes de Dystos-Almyropótamos.

\begin{tabular}{|c|c|c|c|c|c|c|}
\hline \multicolumn{2}{|c|}{ Poljes } & $\begin{array}{c}\text { Longitud } \\
(\mathrm{km})\end{array}$ & $\begin{array}{c}\text { Anchura } \\
\text { máx. }(\mathrm{km})\end{array}$ & Drenaje & Orientación & $\begin{array}{c}\text { Altura del } \\
\text { fondo (m) }\end{array}$ \\
\hline 1 & Krieza & $>6,7$ & 5,1 & Abierto & N-S & 95 \\
\hline 2 & Dystos & 7,7 & 6,3 & Cerrado & NNW-SSE & $18-20$ \\
\hline 3 & Agia Triada & 1,4 & 0,9 & Abierto & N-S & 85 \\
& & $>1,9$ & 0,5 & & NE-SW & 200 \\
\hline 4 & Argyron & 2 & 1,2 & Cerrado & N-S & 40 \\
\hline 5 & Almyropótamos & 4,7 & 1,8 & Abierto & N-S & 60 \\
\hline 6 & Gouba & 2,3 & 0,8 & Abierto & N-S & 200 \\
\hline 7 & Lioutriza & 1,6 & 0,7 & Cerrado & NE-SW & 60 \\
\hline 8 & Prasinon & $>2,8$ & 1,6 & Abierto & NW-SE & N-S \\
\hline 9 & & 2,8 & 1,4 & & NE-SW & 40 \\
\hline 10 & Zarakes & $>1,9$ & 0,4 & Abierto & N-S & 40 \\
\hline 11 & Purgari & $>1,1$ & 1,2 & Abierto & NW-SE & 60 \\
\hline
\end{tabular}




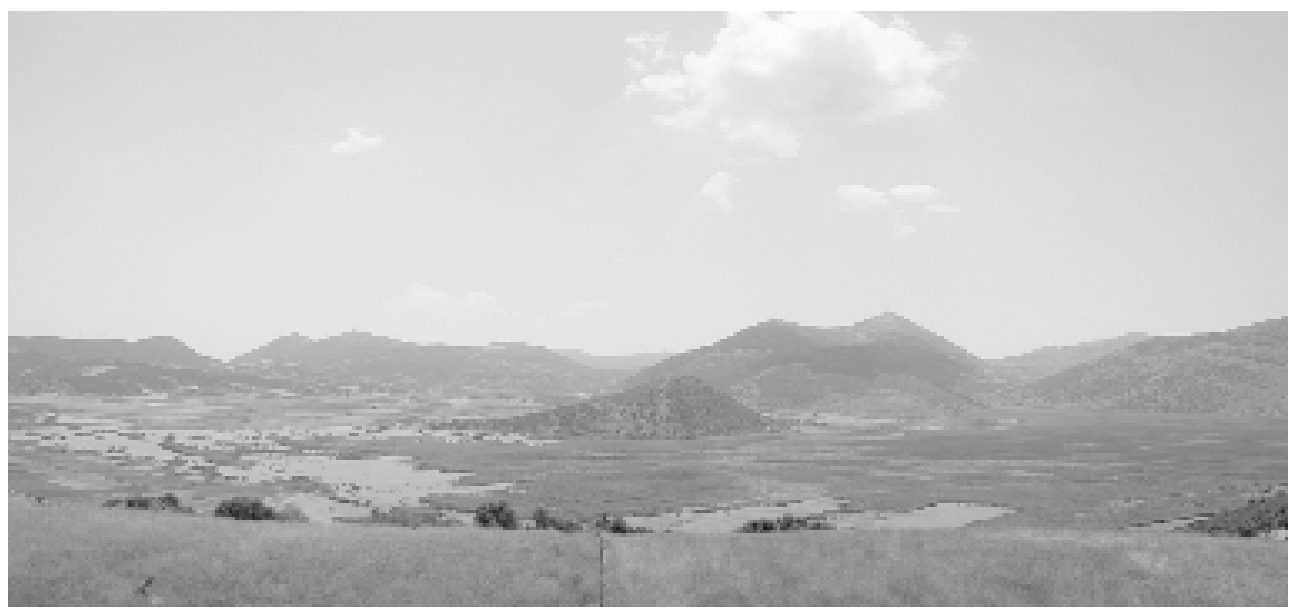

Foto 1. Zona inundada del polje de Dystos y cerro residual (hum) de su fondo.

Entre los poljes aún cerrados destaca el de Dystos por la particularidad de tener una zona endorreica, que puede alcanzar una gran amplitud en momentos de máxima humedad $-4,5 \mathrm{Km}^{2}$ en Octubre de 2003 (Nikolakopoulos (2004)- (Fot. 1). Tanto la importante acción erosiva como el elevado grado de capturas que han sufrido son buenas indicadoras de que se trata de formas de relieve con una larga evolución, hasta el punto de que podemos considerar a algunos de estos poljes como formas residuales y, en todo caso, puede hablarse de "paleopoljes". En territorio continental griego, aunque algunos han sido parcialmente drenados artificialmente desde antiguo, los poljes de Kopais y Helicón (Papadopoulos-Vrynioti, 2004) todavía presentan inundaciones de su fondo en la estación húmeda, lo mismo que muchos de los poljes del karst dinámico (Nicod, 1978) y otros del Mediterráneo sur, como el de Yammoûne (Líbano) (Besançon, 1969), por lo que poseen un gran interés aplicado desde el punto de vista de su aprovechamiento hidrológico.

La planitud del fondo se mantiene de forma generalizada en todo el sistema de poljes con muy pocos relieves residuales, entre los que destaca el bum del polje de Dystos, formando un cerro aislado junto al lago (Fig. 3, Fot. 1); también el polje de Krieza presenta un cerro residual cuya morfología recuerda a un bum. En los márgenes de los poljes la pendiente del fondo aumenta, generándose verdaderos conos rocosos (cônes rocheux) y existiendo rupturas bruscas marginales, ya que se trata de "poljes estructurales", según la clasificación de Ford y Williams (1989).

Las orientaciones de los poljes (Tabla 1) coinciden bastante bien con las líneas estructurales, dominando los rumbos $\mathrm{N}$, NE y NW, lo que significa que hay una 
dependencia directa de las formas fluviokársticas respecto de la red de fracturación y de los accidentes tectónicos principales, como el cabalgamiento del Manto Neohelénico y el eje del anticlinal de Dystos (Figs. 4 y 5). Esta relación con la estructura es muy habitual en el karst mediterráneo (Jennings, 1985; White, 1988), donde el relieve está dirigido claramente por la estructura y especialmente por la actividad neotectónica.

\section{Superficies de aplanamiento y aspectos evolutivos}

Los aplanamientos de origen kárstico aparecen tanto en las superficies altas de los macizos circundantes como en el ámbito interno de los poljes, siendo los elementos geomorfológicos de mayor interés para intentar el establecimiento de la evolución geomorfológica regional.

\subsection{Los aplanamientos cimeros}

Las cumbres de los macizos de composición marmórea con intercalaciones esquistosas, aparecen fuertemente aplanadas (Fot. 2) y karstificadas, de manera que podría interpretarse como el resultado de intensos procesos de karstificación, afectando a amplias superficies de la región analizada.

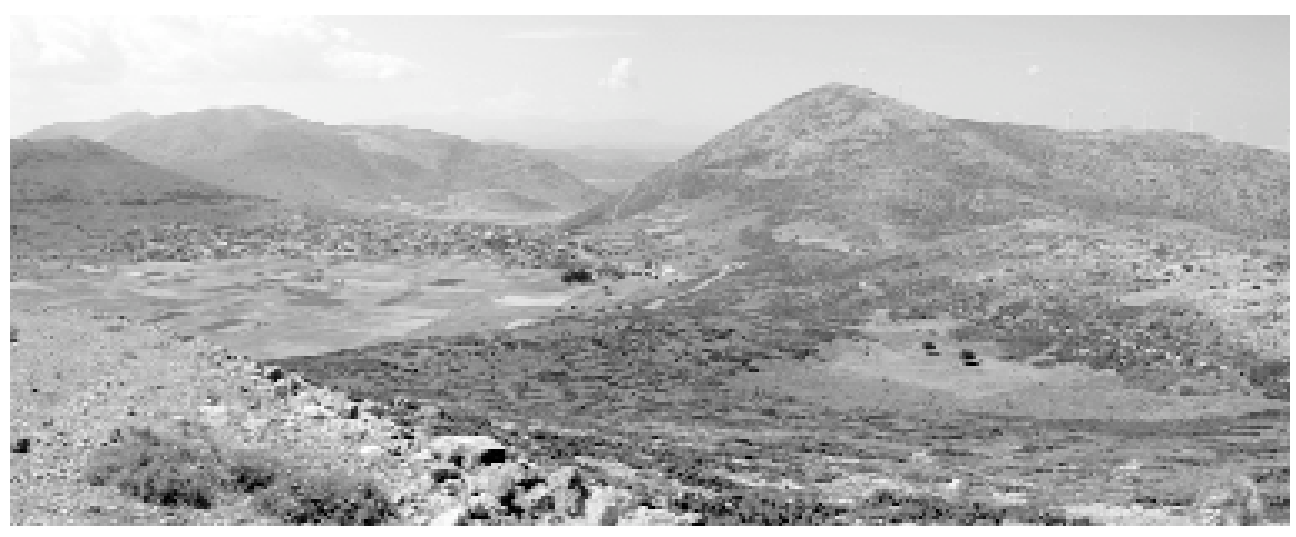

Foto 2. Superficies aplanadas de cumbres que limitan el polje de Argyron (zona cultivada de la izquierda); a la derecha, dolina en embudo. 


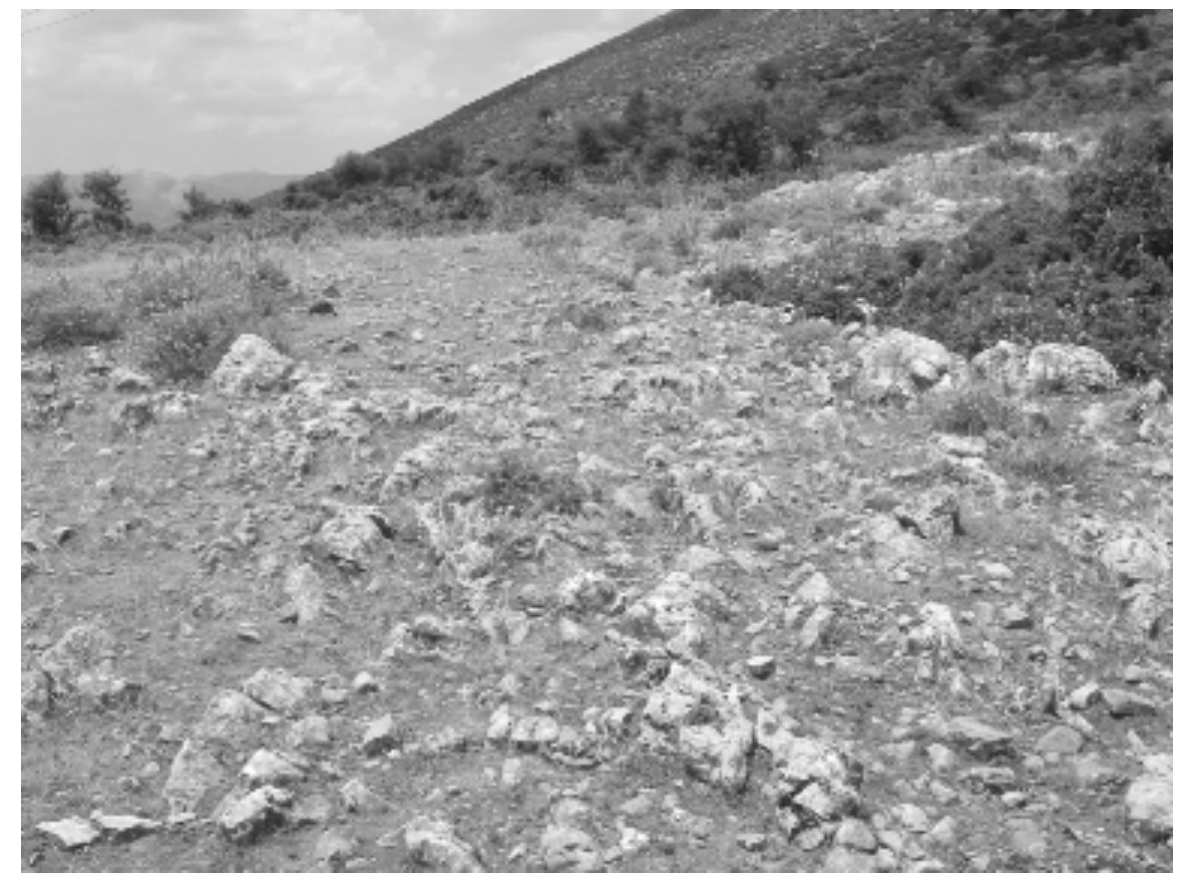

Foto 3. Lapiaces estructurales y oquerosos en superficies de aplanamiento del polje de Prasinon.

Estas superficies forman divisorias convexas entre los valles y poljes con un desarrollo importante del lapiaz y las dolinas. Los lapiaces más abundantes son de tipo estructural y tubular u oqueroso (bohlkarren), con divisorias redondeadas típicas de los lapiaces de origen cubierto (Jennings, 1985; Ford y Williams, 1989), es decir, desarrollados bajo una cubierta edáfica; su actual desnudez se debe a la intensa erosión sufrida por estos paisajes mediterráneos a causa de los seculares incendios y deforestaciones generados por la acción antrópica (Fot. 3).

Las dolinas se extienden en grandes superficies, con gran densidad de formas. Son depresiones de pequeño tamaño, con morfología dominantemente en cubeta o artesa, generadas por procesos de disolución. Por lo general aparecen agrupadas siguiendo alineamientos estructurales y presentan su fondo relleno de materiales arcillosos; son escasos los ejemplos de dolinas profundas, con morfología en embudo, siendo el caso más evidente la existente en el margen oriental del polje de Argyron (Fot. 2).

Estas superficies de aplanamiento de cumbres tienen gran similitud con los llanos kársticos antiguos, señalados por Dufaure $(1975,1985)$ en el Peloponeso, también 
ocupando posición cimera. Sin duda se trata de morfologías heredadas, posiblemente precuaternarias, por lo que pueden considerarse las formas de relieve más antiguas de este sector de la isla de Eubea. Constituirían los restos de antiguas superficies de aplanamiento por corrosión kárstica, deformadas y sobreelevadas por la neotectónica, dando lugar en la actualidad a macizos aislados. En el tercio oriental conforman cuatro unidades montañosas de cumbres aplanadas y fuertemente incididas por los cursos de agua que fluyen hacia el Mar Egeo y hacia los poljes. En el sector occidental componen otros cuatro macizos, aún más extensos, entre los que destaca el de Grosta, en el que alcanzan un gran desarrollo los campos de dolinas (Fig. 3).

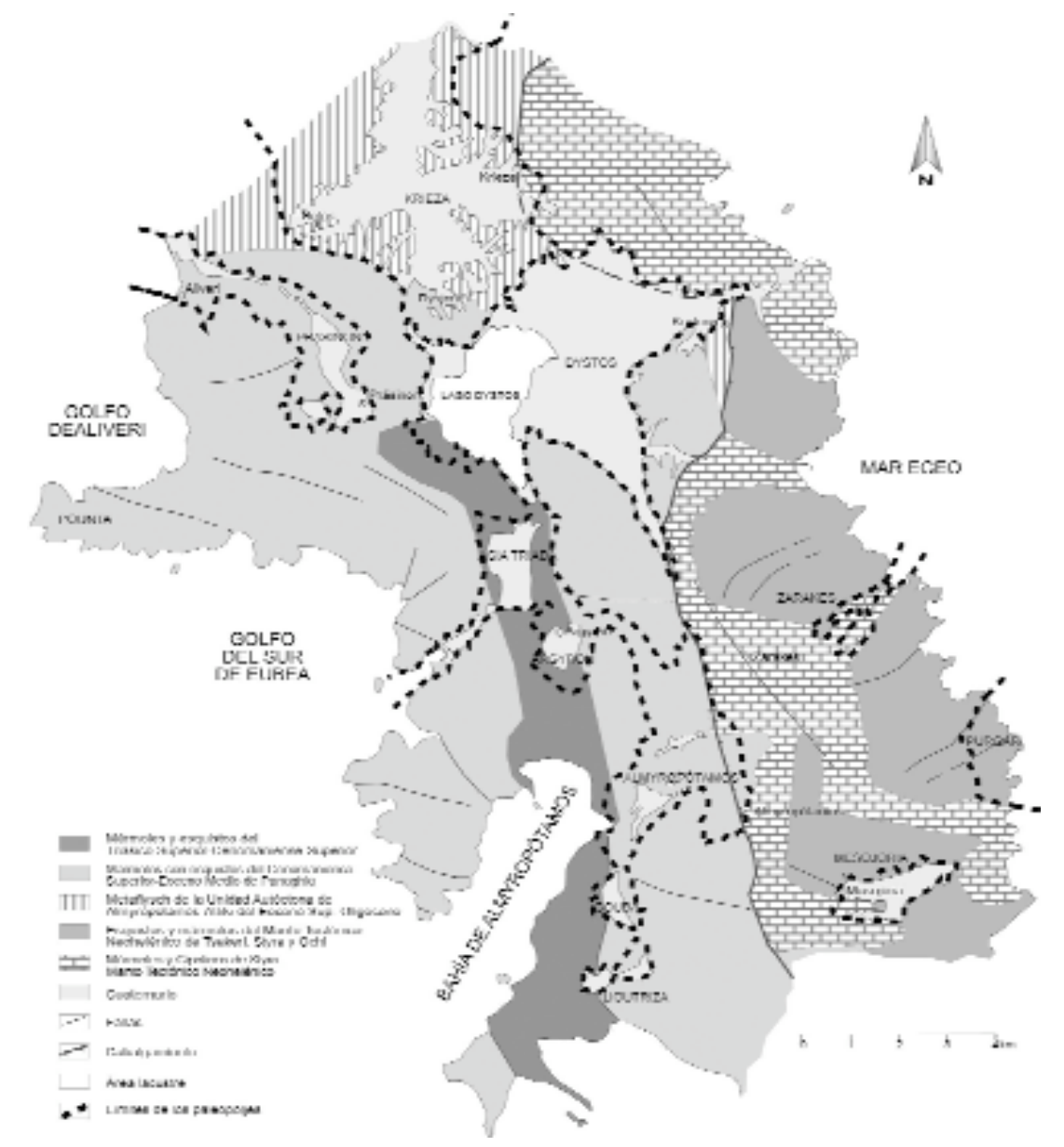

Figura 4. Situación de los poljes y relación con las estructuras geológicas. 


\subsection{Los conos rocosos y las superficies de aplanamiento de los poljes}

En el interior de las depresiones fluviokársticas también se observan superficies erosivas, que corresponden a dos tipologías distintas tal como se ha diferenciado en el mapa geomorfológico: las superficies de criptocorrosión de poljes y los conos rocosos.

Estas dos formas erosivas aparecen aplanando los afloramientos de mármoles y esquistos en la mayoría de los poljes. La erosión posterior ha podido dejar los mármoles o esquistos desnudos o mantener todavía su cubierta de alteración, tal como es señalado para los conos rocosos de Creta por Bonnefont (1985). Parecen representar el resultado de un mismo proceso, en el que la disolución en condiciones de elevada humedad ha favorecido la formación de lapiaces bajo la cubierta de aluviones del fondo del polje, conduciendo al aplanamiento generalizado de esta superficie. Este proceso conocido como criptocorrosión kárstica es citado por diferentes autores en el karst mediterráneo y tropical (Fabre y Nicod, 1982a, b; Nicod, 1985; Salomón et al, 1995) o con la denominación de procesos epikársticos (Papadopoulos-

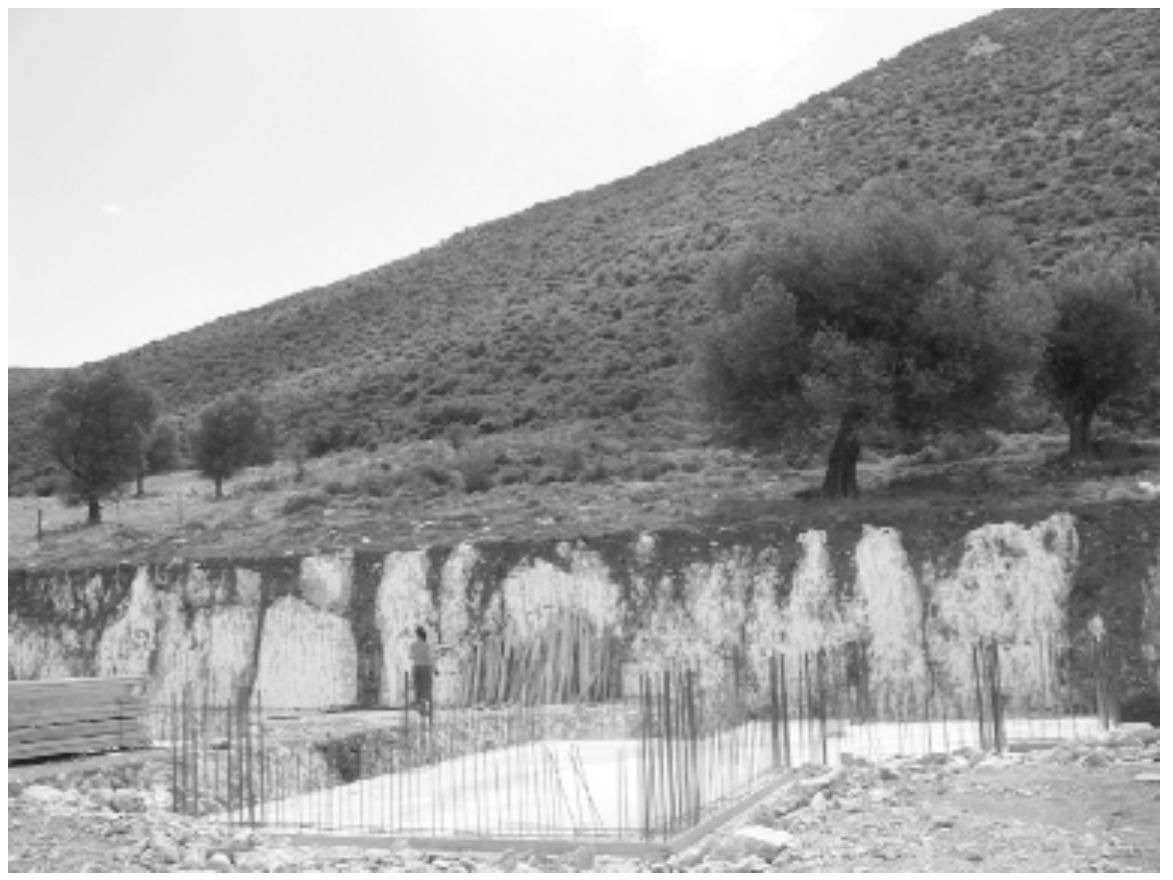

Foto 4. Frente de corrosión kárstica penetrando a través de la red de fracturación de las formaciones marmóreas del polje de Almyopotamos. 


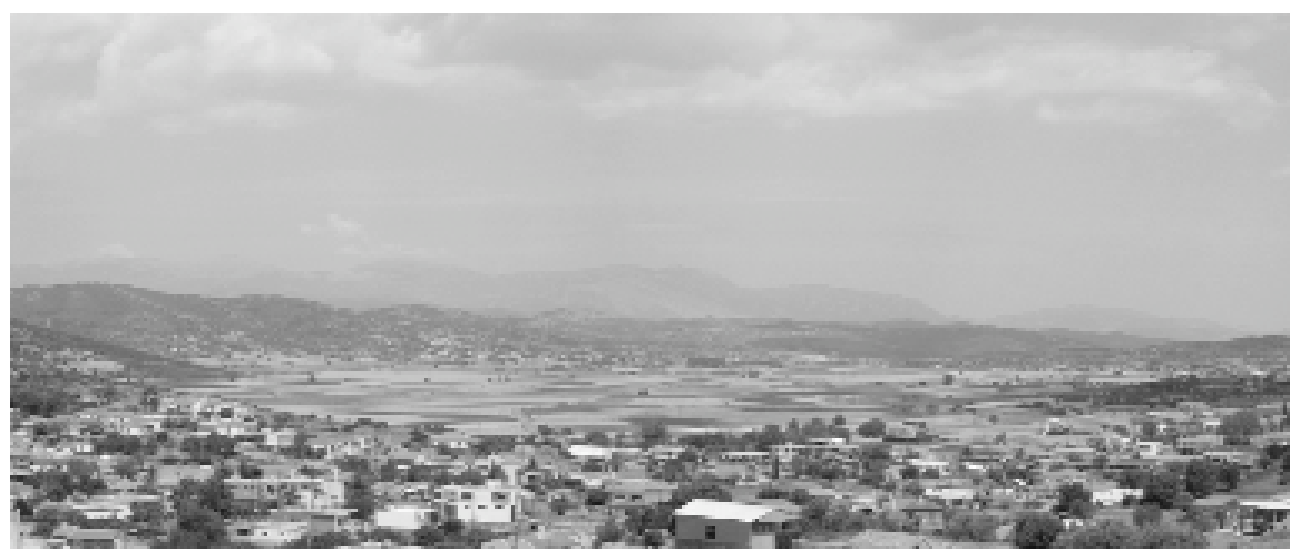

Foto 5. Panorámica del polje de Prasinon.

Vrynioti, 2004). Es explicado como resultado de la acción bioquímica de las raíces de las plantas y los microorganismos a través de las formaciones arcillosas (terra rossa) que componen el fondo del polje, aunque al tratarse de formas heredadas su génesis debe ser interpretada con mucha cautela. Quinif (1999) y Vergaria y Quinif (1997) pusieron en evidencia la diferenciación entre la "corrosion" de la roca desnuda, la "criptoalteración" bajo formaciones no carbonatadas y la "fantômisation" o pseudoendokarst, en el que desaparecen los elementos solubles de la roca pero quedan los menos solubles, como flotando entre la masa alterada. Lo que parece evidente es que los conos rocosos pueden aparecer en diferentes situaciones de humedad y altitudes variadas (Bonnefont, 1985) y es independiente de la importancia de los escarpes a cuyo pie se modelan.

Las superficies antiguas, que han quedado colgadas en ocasiones más de $100 \mathrm{~m}$ respecto al fondo del polje actual, podrían tener el mismo origen que los conos rocosos que se localizan en posiciones más bajas e introduciéndose incluso bajo el fondo del polje funcional.

Las formas de lapiaz más características de las superficies de criptocorrosión son aquellas ligadas a los lapiaces cubiertos, como son las rocas perforadas (kavernösenkarren) de Bögli (1960) o roches perforées de Fabre y Nicod (1982 b) y kluftkarren o fentes corrodées, correspondientes a lapiaz de tipo estructural, de bordes redondeados y rellenos importantes de terra rossa (Fot. 3).

En algunos cortes observados en conos rocosos del polje de Almyropótamos puede apreciarse la fuerte profundización de los frentes de disolución y corrosión 
kárstica en los mármoles del Cenomaniense superior-Eoceno, con abundancia de arcillas de descalcificación rellenando los huecos (Fot. 4). El modelado característico es de lapiaz estructural, con evidente intervención de los sistemas radicales de la vegetación mediterránea.

Los conos rocosos se han localizado en la mayoría de los poljes destacando la gran representación que tienen en el polje de Prasinon (Fot. 5), en el que no existen niveles colgados y el relleno aluvial ha sido desmantelado en la mayor parte del fondo. También en los poljes de Agia Triada, Argyron y Gouba alcanzan un gran desarrollo, formando rampas con escasa cubierta de sedimentos, al existir un importante proceso de lavado como consecuencia de la intensa explotación agrícola y ganadera que han sufrido.

Otro cono rocoso de gran extensión es el que forma el pequeño sector que se conserva del polje de Purgari, que debió ser desmantelado por la evolución del acantilado marino, aunque mantiene plenamente sus características originales, además de dos pequeños fondos de dolina.

Las superficies de aplanamiento de poljes quedan colgadas a diferentes alturas en las zonas marginales de estas depresiones (Fot. 6) y pueden ser interpretadas como antiguos fondos que han quedado aislados de la dinámica actual debido a procesos de encajamiento de los poljes (Gams, 1978).

Las micromorfologías observadas son similares a las señaladas en los conos rocosos, por lo que su génesis podría haber sido exactamente la misma, tal como señala Nicod (1985) para la Basse Provence. Tienen una gran importancia a nivel paleogeográfico, al marcar posiciones altitudinales del paleopolje que pueden permitirnos una aproximación a la reconstrucción de etapas evolutivas cuaternarias, tal como se ha realizado en algunos trabajos sobre los poljes de la Cordillera Ibérica (Gutiérrez et al,

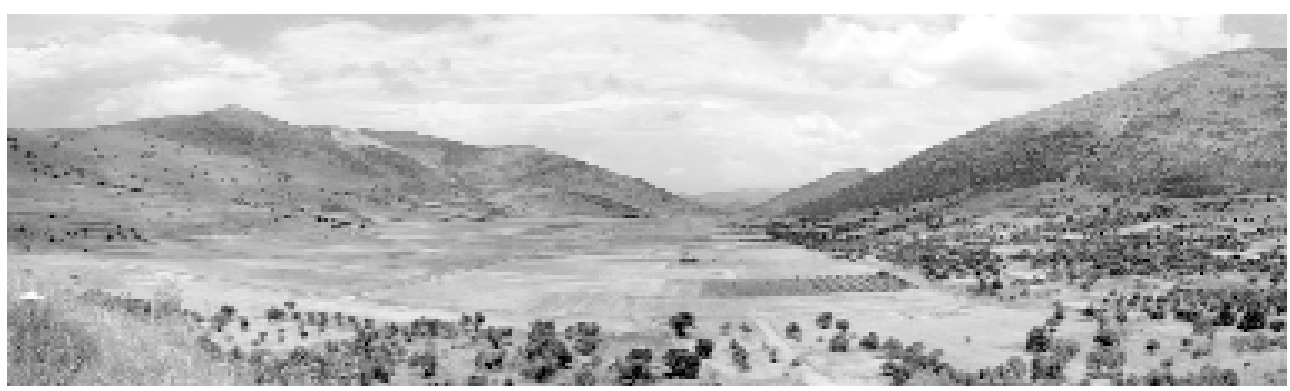

Foto 6. Vista general del polje de Agia Triada, con conos rocosos en su margen oriental (dcha.) y antiguas superficies de aplanamiento en la parte occidental. 
Tabla 2. Altitudes (m.s.n.m.) de los niveles de aplanamiento de los poljes.

\begin{tabular}{|c|c|c|c|c|}
\hline & Argyron & Agia triada & Dystos & Krieza \\
\hline 20 & & & $\mathrm{X}$ & \\
\hline 40 & & & $\mathrm{X}$ & \\
\hline 80 & & & $\mathrm{X}$ & $\mathrm{X}$ \\
\hline 85 & & $\mathrm{X}$ & & \\
\hline 100 & & $\bar{X}$ & & $\mathrm{X}$ \\
\hline 120 & & $\mathrm{X}$ & & $\mathrm{X}$ \\
\hline 140 & & $\bar{X}$ & $\mathrm{X}$ & $\bar{X}$ \\
\hline 180 & & & $\mathrm{X}$ & \\
\hline 200 & $\mathrm{X}$ & & & \\
\hline 210 & $\mathrm{X}$ & & & \\
\hline 220 & $\mathrm{X}$ & & & \\
\hline 240 & $\mathrm{X}$ & & & \\
\hline
\end{tabular}

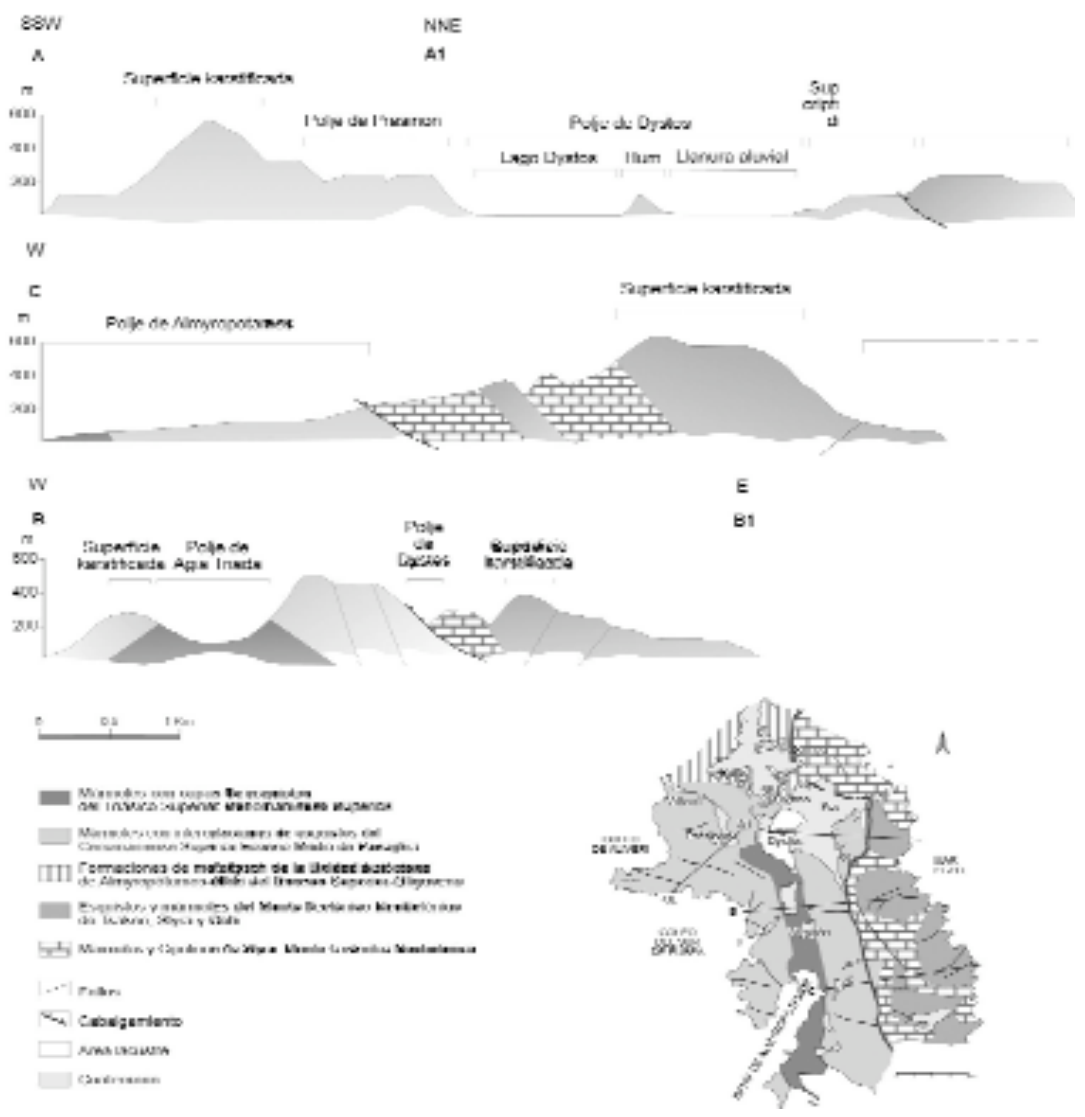

Figura 5. Cortes geomorfológicos a través de los diferentes poljes. 
1982; Peña et al, 1989). No se ha apreciado una equivalencia total en las alturas relativas de los retazos de superficies de aplanamiento conservadas, sino que como puede apreciarse en la Tabla 2 y en la Fig. 5, cada polje presenta su particular organización de niveles aunque en algunos casos hay coincidencias notables en las alturas.

Así, por ejemplo, las alturas observadas en los poljes de Krieza y Dystos coinciden bastante bien con las señaladas en el de Agia Triada, lo que podría llevar a la deducción de una evolución conjunta para estos tres poljes durante las etapas antiguas hasta su diferenciación definitiva en época más reciente. Sin embargo el polje de Argyron, con alturas totalmente distintas, podría haber evolucionado siempre de forma independiente. Algunos poljes presentan un menor desarrollo de estas superficies, como los de Prasinon, Gouba, Lioutriza y Mesojoria.

\section{Conclusiones}

La cartografía geomorfológica del sistema de poljes de Dystos-Almyropótamos ha permitido la diferenciación de once unidades kársticas de tipo polje separadas por relieves montañosos que asimismo presentan superficies cimeras debidas a la karstificación. Tanto las litologías, con una gran abundancia de afloramientos de mármoles, como la estructura, con la existencia de unas líneas tectónicas bien definidas, en las que predominan las direcciones Norte-Sur, han influido de manera decisiva en la configuración de esta importante zona kárstica. A esta influencia lito-estructural hay que añadir la evolución paleoclimática cuaternaria, con fases favorables al desarrollo de estos procesos.

Las superficies de aplanamiento que afectan a las formaciones carbonatadas comenzaron a generarse seguramente con anterioridad al Cuaternario, posiblemente durante el Mioceno superior, como señala Dufaure (1985) para aplanamientos kársticos en el Peloponeso; los restos de estas superficies antiguas se conservan en las cumbres de los macizos montañosos, acompañados de campos de lapiaz y de dolinas. Estas superficies de erosión posiblemente constituyeron un conjunto único, que luego quedaría formando unidades aisladas debido a importantes reactivaciones tectónicas, basculando diferentes bloques y generando el rehundimiento de otras zonas a partir de accidentes tectónicos distensivos. Muchas de estas zonas deformadas podrían ser el origen inicial de algunos poljes, que habría que clasificarlos como "estructurales", Sin embargo, las evidencias demostrativas de este evento y la posibilidad de situarlo cronológicamente son prácticamente nulas.

Ya con una cronología seguramente cuaternaria se han modelado los aplanamientos internos de los poljes, que responden a procesos de una gran similitud, gene- 
rados como un karst cubierto con gran influencia de los procesos bioquímicos. La historia del funcionamiento del polje está registrada en estos aplanamientos laterales, aunque su asignación cronológica es difícil de establecer por las dificultades de datación.

Los diferentes niveles de aplanamiento localizados tienen cierta similitud con los analizados en la Cordillera Ibérica, donde fueron definidos como de tipo ibérico, muy extendidos en las sierras de Gúdar-Maestrazgo (Lozano 1988), Javalambre (Gutiérrez et al, 1983), Albarracín (Peña et al, 1989) y Cuenca (Alonso et al, 1987). Sin embargo, frente al perfecto escalonamiento que presentan las superficies de aplanamiento de los poljes ibéricos, de gran utilidad para su reconstrucción evolutiva, los poljes analizados en la isla de Eubea no presentan una organización tan regular de los aplanamientos, por lo que las conclusiones evolutivas no resultan tan evidentes. Aún así, como ya hemos indicado, hay algunas correspondencias entre los niveles de estos poljes, que podrían tomarse como indicadores de evoluciones conjuntas entre algunas de las depresiones en ciertos momentos de su evolución.

Los procesos de aplanamiento y su alternancia con etapas de incisión suele interpretarse como consecuencia de la existencia de fases climáticas contrastadas favorables en algunos casos a la disolución de tipo lateral (etapas húmedas) y en otros a la concentración del drenaje de forma simultánea con la profundización de la red fluvial, con una menor importancia de la disolución y predominio de la acción fluvial (etapas más secas), como fue señalado por Nicod (1967) para los poljes de la Basse Provence. Por otra parte, han podido jugar un papel importante en los procesos de incisión las reactivaciones neotectónicas producidas durante el Cuaternario, ya que la isla de Eubea forma parte de unidades geológicas todavía afectadas por fuerte actividad sísmica y fallas activas.

\section{Agradecimientos}

Este trabajo se ha realizado en el marco del proyecto de investigación Global Change project GC/02/027 The Degradation of the Environment and more specifically the Desertification, in the Past, Present and Future y dentro de las actividades del Grupo de Investigación del Gobierno de Aragón PALEOQ. Agradecemos las aportaciones y observaciones de los revisores del artículo. 


\section{Bibliografía}

Alonso, F., González, J. A. y Ugarte, F. (1987) Sur l'evolution de trois grandes dèpressions karstiques de la Serranía de Cuenca (Spagne). Mèditerranée 1, 21-32.

Andronopoulos, G. (dir.) (1991) Geological map of Greece 1:50.000, Aliveri sheet. IGME, Atenas.

Besançon, J. (1969) Note sur l’hydrologie du polje de Yammoûne. Méditerranée, 2, 143163.

Bögli, A. (1960) Kalklösung und karrenbildung. Z. Geomorph. Suppl.2, 4-21.

Bonnefont., J.C. (1972) La Crète. Étude géomorphologique. Thèse Doctorat Univ. de Lille, $845 \mathrm{p}$.

Bonnefont, J.C. (1985) Observations sur els cônes rocheux de lîle de Crète. En Cônes Rocheux, Mémoires. et Documents de Géographie, 53-65. CNRS. Paris.

Dufaure, J.J. (1975) Le relief du Péloponnèse. Thèse Univ. Paris Sorbonne, 1422 p.

Dufaure, J. J. (1985) Plans, pédiments et cônes rocheux calcaires du Péloponnèse (Grèce). Mémoires et documents de Géographie, 73-99. C.N.R.S., París.

Fabre, G. y Nicod, J. (1982 a) Modalités et rôle de la corrosion crypto-karstique dans les karsts mèditerranéens et tropicaux. $Z$. Geomorph. N. F., 26 (2), pp. 209-224.

Fabre, G. y Nicod, J. (1982 b) Lapiés couverts, modalitées et rôle de la corrosion cryptokarstique. En Nicod, J. (Ed.): Phénoménes karstiques III, Mémoires et documents du C.N.R.S., pp.115-117.

Faugères, L. (1978) Recherches géomorphologiques en Grèce septentrionale: Macedonie centrale et occidentale. Thèse Doctorat Univ. de Lille.

Ford D. y Williams, P. (1989) Karst
Geomorphology and Hydrology. Unwin Hyman. 601 p.

Gams, I. (1978) The polje: the problem of definition, Z. Geomorph. N. F. 22 (2), 170181.

Gèze, B. (1973) Lexique des termes français de spèléologie physique et de karstologie. Annales de spéléologie, 28, 1-20.

Gutiérrez, M. Peña, J. L. y Simón J. L. (1982) El polje de Vistabella del Maestrazgo (Provincia de Castellón). Reunión monográfica sobre el karst-Larra 82, 95-104.

Gutiérrez, M. Peña, J. L. y Simón J. L. (1983) Los poljes de la región de TorrijasAbejuela (prov. de Teruel, Castellón y Valencia), Teruel, 69, 99-108.

Jennings, J. N. (1985) Karst Geomorphology. Blackwell. 293 p

Lozano, M. V. (1988) Los poljes de la región de Mosqueruela (Serranías de GúdarMaestrazgo, provincia de Teruel). Cuaternario y Geomorfología, 2, 57-66.

Monroe, W. H. (1970) A glosary of karst terminology. Geol. Survey Water Supply Paper, 1899-K, 1-26.

Nicod, J. (1967) Recherches morphologiques en Base-Provence calcaire. Thése doct, univ. Aix-en-Provence.

Nicod, J. (1978) Les eaux et l'amenagement des poljes du karst dinarique. Méditerranée, 32(1-2), 85-104.

Nicod, J.(1985) Les cônes rocheux en Provence et dans quelques régions de comparaison. Rapports avec les surfaces de corrosion des poljés. En Cônes Rocheux, Mémoires et Documents de Géographie, 107-117. CNRS. Paris.

Nikolakopoulos, K., y Pavlopoulos, K. (2004) Environmental Monitoring Of Lake Dystos 
In Central Evia, Greece Using Multitemporal And Multisensor Remote Sensing Data, Remote Sensing for Agriculture, Ecosystems, and Hydrology, VI.

Papadopoulos-Vrynioti， K. (1998-1999) Karst landform of Lichas Peninsula (NW of Euboea-Greece). Theoretical and Applied Karstology, 11-12, 53.56.

Papadopoulos-Vrynioti, K. (2004) The role of epikarst in the morphogenese of the karstic forms in Greece and specially of the karstic hollow forms. Acta Carsologica, 33/1, 14, 219-235. Liubljana.

Papadopoulos-Vrynioti, K. \& Kirdis, S. (1998) Typical dinaric karst in Central Euboea. Bull. Geogr. Soc. Greece, 218-222. Atenas.

Peña, J.L., Jiménez, A y Echeverría, M.T. (1989) Geomorphological cartography and evolutionary aspects of the Sierra de Albarracín poljes (Eastern Iberian Ranges, Teruel, Spain), Geografía Física e Dinámica Quaternaria, 12, 51-57.

Quinif, Y. (1999) Fantômisation, criptoalteration et alteration sur roche nue. Le triptyque de la karstification. Actes Coll. Karst 99. Grandes Causses-Vercors, 159-164.

Salomon, J.N., Pomel, S. y Nicod, J. (1995) L'évolution des cryptokarts: comparaision entre le Périgord-Quercy et le Franjen Alb (Allemagne). Z. Geomorph., N.F., 39(4), 381-409.

Vergaria, A. y Quinif, Y. (1997) Les paléokarts du Hainaut. Geodynamica Acta, 10-4, 175187.

White, N.B. (1988) Geomorphology and Hydrology of karsts terrains. Oxford Univ. Press, $464 \mathrm{p}$. 